\title{
An Up to 20-Year Retrospective Study of 4-Unit Fixed Dental Prostheses for the Replacement of 2 Missing Adjacent Teeth
}

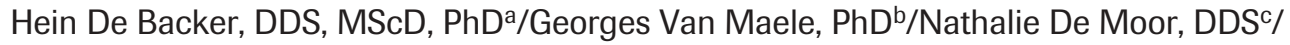

Linda Van den Berghe, DDS, MScD, $\mathrm{PhD}^{\mathrm{d}}$

\begin{abstract}
Purpose: This study evaluated treatment outcomes of 4-unit porcelain-fused-to-gold fixed dental prostheses (FDPs) replacing 2 adjacent missing teeth. Materials and Methods: A total of 102 FDPs made in an undergraduate university clinic for 73 patients were evaluated for up to 20 years, with a mean survival follow-up time of 11.4 years. All patients were offered an oral health maintenance program. Treatment failures were divided into irreversible (loss of FDPs/finish line involved) or reversible (FDPs and finish line intact after conservative treatment) complications and into biologic and technical/patient-related failures. Results: The Kaplan-Meier overall estimated survival rate was $68.3 \%$ at year 20 . There was a statistically significant difference $(P=.007)$ between the survival rates in the maxilla for the vital group $(73.8 \%)$ and those for the root canal-treated group (25.1\%). Comparing the survival rate in the root canal-treated group for the restorations in the maxilla (25.1\%) versus the mandible $(66.8 \%)$, a statistically significant difference $(P=.011)$ was found. The main reason for irreversible failure was caries (32.0\%). Conclusion: The estimated successful outcome of 4-unit FDPs over an up to 20-year period is considered favorable and should be compared with the survival rates of other treatment options for the replacement of 2 adjacent teeth. Occurrence of a previously reversible complication appears to be a predictive factor for an irreversible complication later on. A reversible complication within the first 2 years will lead to an early irreversible complication. Int J Prosthodont 2008;21:259-266.
\end{abstract}

$A^{\prime}$ variety of treatment options are available to replace missing teeth. Conventional metal-ceramic fixed dental prostheses (FDPs), removable partial dentures, resin-bonded FDPs, implant-supported full crowns, or FDPs and orthodontic therapy are feasible treatment options. An implant-supported FDP is currently considered as the primary treatment for the reconstruction of a single tooth diastema. However, appropriate treatment planning is dependent on each individual clini-

\footnotetext{
aSenior Researcher, Centre for Special Care, PaeCaMed Research, Unit of Gnathology and Temporomandibular Disorders, Dental School, Faculty of Medicine and Health Sciences, Ghent University, Belgium.

bProfessor, Department of Medical Statistics, Faculty of Medicine and Health Sciences, Ghent University, Belgium.

cPrivate Practice, Ghent, Belgium.

dProfessor, Centre for Special Care, PaeCaMed Research, Unit of Gnathology and Temporomandibular Disorders, Dental School, Faculty of Medicine and Health Sciences, Ghent University, Belgium.
}

Correspondence to: Dr Hein De Backer, Hulststraat 8, 8700 Tielt, Belgium. E-mail: hein.debacker@skynet.be cal situation. The patient's expectations, general health conditions, and socioeconomic profile are important parameters that must be considered.

The loss of 2 adjacent teeth will alter the gingival appearance, adjacent periodontal tissues, and underlying bone. This will almost invariably lead to loss of the original scalloped configuration of the supporting tissues and may pose an esthetic prosthodontic challenge. However, this can be addressed efficiently with pontics that are well adapted to the edentulous ridge, thereby creating the illusion of a scalloped gingival continuum with distinctly formed interproximal papillae. The use of implants to support a traditionally designed FDP may make the reestablishment of naturally appealing esthetics more complex. ${ }^{1-3}$ Depending on the case, soft tissue augmentation techniques may help achieve a respectable esthetic result., ${ }^{4,5}$ Provisional restorations with either abutment-support technique are important adjunctive aids in planning esthetic treatment outcomes since they offer a controlled pontic design during the healing process in a protocol for soft tissue management techniques. ${ }^{6}$ 
Most studies on the life span of fixed prosthetic restorations are retrospective and tend to be divided into short- and long-term clinical evaluations, with the latter rather limited in number. ${ }^{7}$ Studies on implant-supported or resin-bonded prostheses are, however, much better documented. ${ }^{7,8}$ Results on 4-unit FDPs or shortspan FDPs (3 or 4 units) tend to be reported as part of a broader survey, ${ }^{9-14}$ with scarce and limited statistics on functional life spans or comments on failed FDPs.

In general, it is difficult to compare the data on the survival of FDPs. ${ }^{11,12,15-23}$ The most substantial differences between studies are found in the definition of failure, the population groups studied, the need for standardization of the terminology, and the consequent use of scientific rules in the design of the studies. ${ }^{24-26}$

The aim of the present study was to assess the treatment outcome of conventional 4-unit porcelain-fusedto-gold FDPs, replacing 2 missing adjacent teeth, to determine frequencies and causes of failures, and to evaluate the outcome of the use of post-and-core abutments in 4-unit FDPs compared to vital abutments.

\section{Materials and Methods}

\section{Materials}

A total of 126 four-unit FDPs was made over a period of 18 years, between 1974 and 1992, in the undergraduate clinic of the former Department of Fixed Prosthodontics and Periodontology, Ghent University, Belgium. Cantilever FDPs were not included in the present study. This group of 4-unit FDPs was part of a larger study group of FDPs at the same department. ${ }^{15}$

For the current study, complete treatment and follow-up records of 73 patients $(70.6 \%$ women and $29.4 \%$ men) with a mean age of 63.6 years (range: 37.0 to 94.2 years) and 102 FDPs were available for analysis, representing $81 \%$ of the total number of 4-unit FDPs made during that period. The dropout rate of $19 \%$ was the result of the following reasons: patients chose a private practitioner for maintenance, moved to another city, could not be traced, or died during the follow-up period. None of the patients in the dropout group were contacted by telephone and no questionnaires were sent either to these patients or to their former or current clinicians to collect supplementary information.

The FDPs consisted of porcelain-fused-to-gold or gold retainers. Retainers in the anterior region were always covered with porcelain. Retainers on molars were gold or porcelain-fused-to-gold restorations, depending on the esthetic choice of the patient or the technical preference of the practitioner. In the posterior region, most retainers had a supragingivally located margin. For esthetic reasons, the retainer margin in the anterior region was located at the gingival margin. For the post-and-core abutment teeth, a standard ferrule of $2 \mathrm{~mm}$ was preferred-though many teeth had a limited ferrule-and at least $10 \mathrm{~mm}$ (range: 7 to $15 \mathrm{~mm}$ ) of the root canal filling was removed. No special root canal preparation was used, with care taken to avoid excessive removal of dentin substance. No direct restorative techniques, special burs with prefabricated posts, or additional parapulpal pins were used. The impression of the abutment tooth was made with a polyether material (Impregum, 3M ESPE). For the root canal, the polyether material was inserted with the help of a lentulo, but no other devices such as burnout posts were used. All cast gold posts and cores were made separately from their retainer of the same gold alloys (type IV, Degudent U, Degussa) used for the retainers and pontics. All retentive surfaces of the restorations were sandblasted $(50 \mu \mathrm{m})$ during the last laboratory phase prior to cementation. All 4-unit FDPs were cemented with zinc phosphate cement (Harvard, Richmond Harvard) under the same strict conditions. This project (EC UZG 2005/100) was approved by the Ethics Committee, OG 017, University Hospital, Ghent, Belgium.

\section{Methods}

Before starting prosthetic treatment, all patients underwent periodontal screening. Patients without periodontal problems were treated immediately with FDPs. Periodontally affected patients were scheduled for periodontal treatment followed by a controlled oral hygiene program. At reevaluation 6 months after scaling and root planing, the decision for prosthetic treatment or the need for further periodontal treatment was made. Some of the patients had FDP treatment 1 year after periodontal treatment. The data at the first recall visit after cementation (within 1 month) were used as baseline and had to be confirmed with the data at the first screening for patients without periodontal problems and at 6 - or 12-month control visits after periodontal treatment for the periodontally affected patients. All patients were invited and agreed to participate in a regular supportive maintenance program every 6 months. ${ }^{15} \mathrm{~A}$ number of patients interrupted this program or preferred to visit a private practitioner. Table 1 presents the compliance with recall at the prosthetic restoration level. During these maintenance sessions, a number of diagnostic and therapeutic steps were undertaken: whole mouth plaque score after staining with a dichotomous reading, bleeding on gentle probing of the gingival sulcus, periapical radiographs, recording of new caries lesions or secondary caries, control of the retention of the restoration, and recording of mechanical failures. Probing depth at 6 or 8 sites per tooth was recorded using a Michigan periodontal probe. At each session, patients were rein- 
Table 1 Recall Frequency for the 4-Unit FDPs

\begin{tabular}{lcrrr}
\hline Visits per year & $\begin{array}{c}\text { No. } \\
\text { surviving }\end{array}$ & $\begin{array}{c}\text { No. } \\
\text { failed }\end{array}$ & $\begin{array}{c}\% \\
\text { surviving }\end{array}$ & $\begin{array}{c}\% \\
\text { failed }\end{array}$ \\
\hline$\geq 2$ & 14 & 11 & 18.2 & 44.0 \\
1 & 30 & 6 & 39.0 & 24.0 \\
$<1$ & 15 & 2 & 19.5 & 8.0 \\
No recall & 4 & 5 & 5.2 & 20.0 \\
Private practice & 14 & 1 & 18.2 & 4.0 \\
Total & 77 & 25 & 100.0 & 100.0 \\
\hline
\end{tabular}

Table 2 Distribution of 4-Unit FDPs in the Maxilla and Mandible for the Vital and RCT Groups

\begin{tabular}{lccr}
\hline & Vital group (\%) & RCT group (\%) & Total (\%) \\
\hline Maxilla & $20(55.5)$ & $16(44.5)$ & $36(35.3)$ \\
Mandible & $22(33.5)$ & $44(66.5)$ & $66(64.7)$ \\
Total & $42(41.2)$ & $60(59.8)$ & $102(100)$ \\
\hline
\end{tabular}

Fig 1 Frequency distributions for the survival times in the 4-unit FDPs.

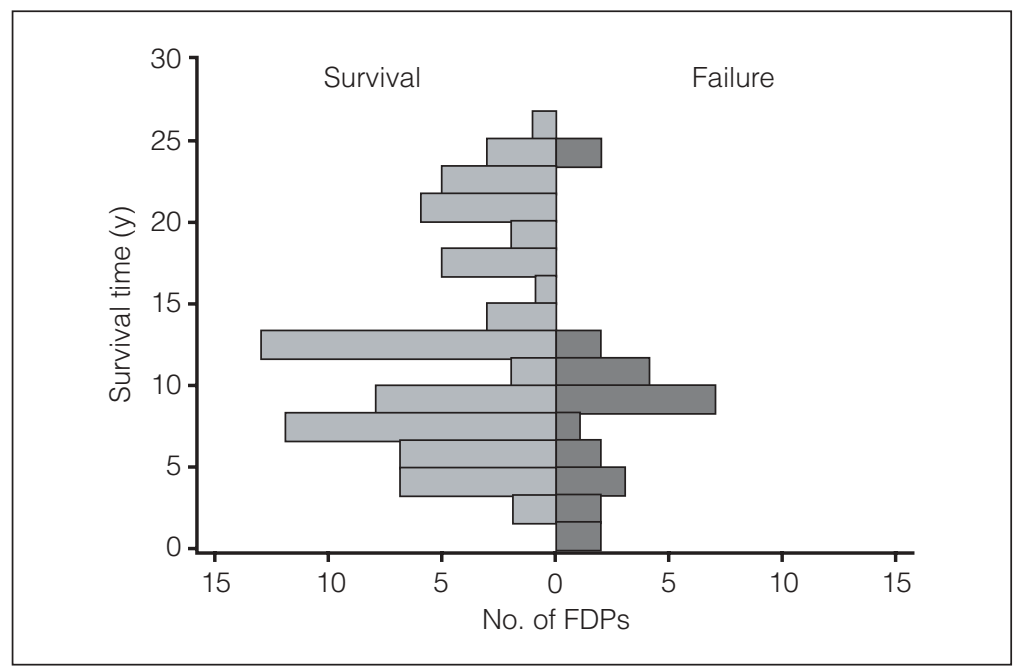

structed in plaque control. If the interdental morphology allowed, instructions on cleaning with interproximal brushes or superfloss were given. At each session, plaque and supra- and subgingival calculus were removed. Patients were scheduled for scaling and root planing when periodontally indicated. The patients who interrupted this program or preferred to visit a private practitioner were sent an invitation by mail on a single occasion for a free checkup. During this last evaluation session, the aforementioned diagnostic and therapeutic steps were undertaken, thus reintegrating this patient group into the present study results. Failures were divided into biologic or technical/patient-related failures and into reversible or irreversible complications. Caries, periodontal problems, fracture of the abutment tooth, and endodontic problems were considered biologic failures. Loss of retention and fracture of the framework were considered technical/patient-related failures. Failures were defined as irreversible complications if the FDP or a tooth was lost or the finish line was involved and as reversible complications if recementation was needed after loss of retention or endodontic treatment/filling on an abutment tooth with the FDP and the finish line still intact. An FDP may have had a reversible complication but ended up in the surviving group at the final evaluation or may have had a reversible complication followed by an irreversible complication, thus ending up in the failure group.

\section{Statistical Analysis}

The survival estimation method of Kaplan and Meier with a 95\% confidence interval (Cl) was used. ${ }^{27}$ The log-rank test was used to determine whether some survival functions differed between groups. ${ }^{28}$ Statistical significance of differences was calculated using the chi-square test (Fisher exact test). The significance level was set at $\alpha=.05$.

\section{Results}

\section{Descriptive Data}

Frequency distributions for the survival times from 0 to 26 years of the investigated 4-unit FDPs are shown in Fig 1 in relation to survival versus failure. This symmetric graph reveals a well-populated study group up to 24 years. The mean survival follow-up time was 11.4 years.

The distribution of 4-unit FDPs in the maxilla and mandible and in the vital and root canal-treated (RCT) groups is presented in Table 2. The mean tooth units per FDP was 4.0, and the pontic/abutment ratio was 1.0 (204 retainers and 204 pontics). The antagonist was the natural dentition in $57.1 \%$ of the patients, an FDP in $37.8 \%$, and a complete denture or edentulous space in $5.1 \%$. 
Table 3 Frequency Distribution of 4-Unit FDPs Per Patient and the No. of 4-Unit FDPs Failed or Surviving Within the Same Patient

\begin{tabular}{lcc}
\hline FDP/patient & No. failed & No. of patients \\
\hline $1(72.7 \%)$ & 0 & 39 \\
$2(17.8 \%)$ & 1 & 14 \\
& 0 & 8 \\
$3(6.8 \%)$ & 1 & 1 \\
& 2 & 2 \\
$4(2.7 \%)$ & 0 & 3 \\
& 1 & 1 \\
& 3 & 2 \\
\hline
\end{tabular}

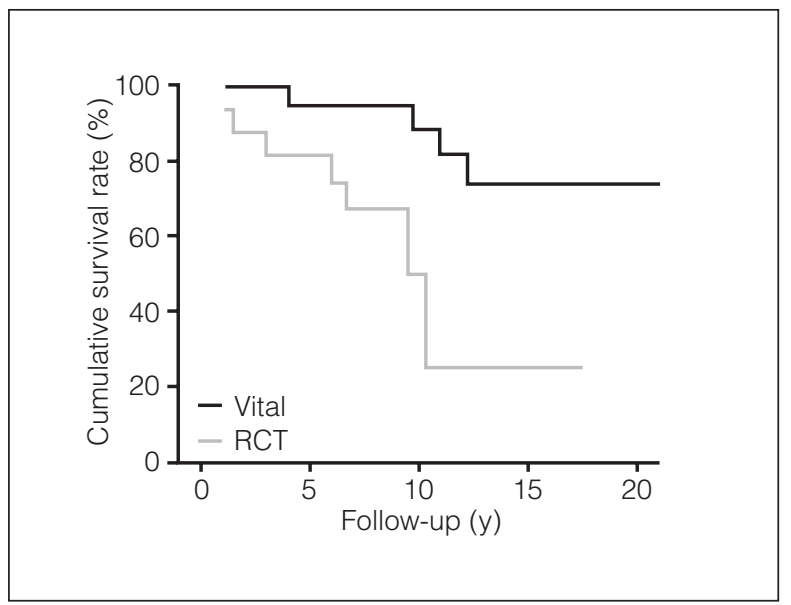

Fig 3 Kaplan-Meier survival curves for the vital and RCT groups in the maxilla $(P=.007)$.

Table 3 shows the number of 4-unit FDPs placed per patient, the number of failed or surviving restorations within the patient, and the frequency distribution in the study population. In this investigated group, $24.5 \%$ $(n=25)$ of the FDPs failed. One patient (4.8\%) had 3 failing FDPs, 2 patients (9.5\%) had 2 failing FDPs, while 18 patients (85.7\%) had only 1 failing FDP, representing $28.8 \%$ of the total patients. Complications were not more likely in any particular patient.

\section{Kaplan-Meier Survival Curves}

The Kaplan-Meier survival estimation for all 4-unit FDPs was 92.9\% (95\% Cl: 88\%-98\%) at year 5, 78.7\% $(69 \%-88 \%)$ at year 10 , and $68.3 \%(57 \%-79 \%)$ at years 15 and 20.

For the vital and RCT groups, Figs 2 to 4 show the Kaplan-Meier survival curves overall and for the maxilla and mandible. For the overall estimation (Fig 2),

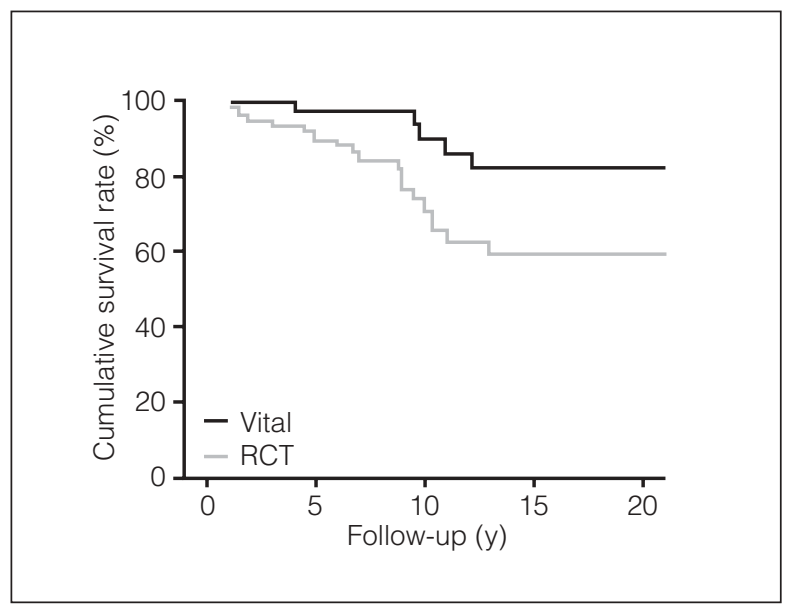

Fig 2 Kaplan-Meier survival curves for the vital and RCT groups overall $(P=.044)$

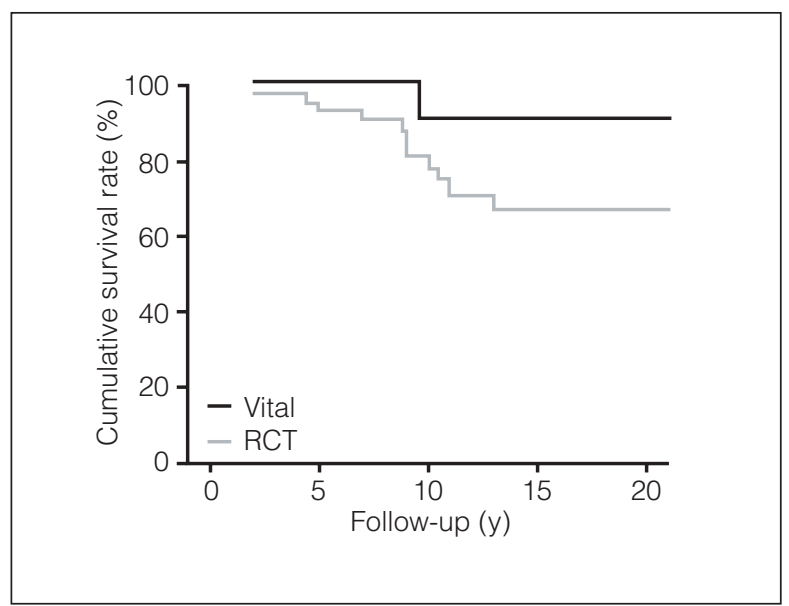

Fig 4 Kaplan-Meier survival curves for the vital and RCT groups in the mandible $(P=.092)$.

there was a significant difference (log-rank $P=.044)$ between the vital and RCT groups at year 20 . The survival estimation for the vital group was $97.6 \%$ $(93 \%-100 \%)$ at year $5,89.9 \%(79 \%-100 \%)$ at year 10 , and $81.9 \%(67 \%-97 \%)$ at years 15 and 20 . For the RCT group, the survival estimate was $89.8 \%(82 \%-98 \%)$ at year $5,71.0 \%(58 \%-84 \%)$ at year 10 , and $59.4 \%$ $(44 \%-75 \%)$ at years 15 and 20 .

Regarding the survival estimates for the maxilla (Fig 3 ), there was a highly statistically significant difference between the groups $(P=.007)$. The survival estimation for the vital group in the maxilla was $94.7 \%$ $(85 \%-100 \%)$ at year $5,88.0 \%(72 \%-100 \%)$ at year 10 , and $73.8 \%(51 \%-96 \%)$ at years 15 and 20 . For the RCT group in the maxilla, the survival estimation was $81.3 \%$ $(62 \%-100 \%)$ at year $5,50.3 \%(17 \%-84 \%)$ at years 10 and 15 , and $25.1 \%(0 \%-64 \%)$ at year 17 . Regarding the survival estimation for the mandible (Fig 4), there was no significant difference $(P=.092)$ between the groups. 


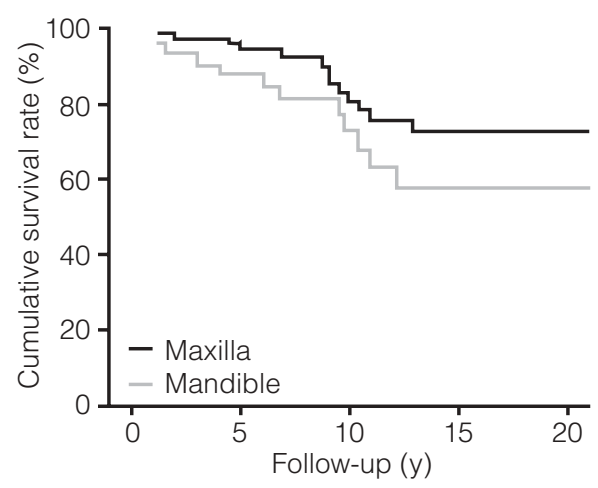

Fig 5 Kaplan-Meier survival curves for the maxilla and mandible overall $(P=.097)$.

The survival estimation for the vital group in the mandible was $100 \%$ at year 5 and $90.9 \%$ (74\%-100\%) at years 10, 15, and 20. For the RCT group in the mandible, the survival estimate was $93.0 \%$ (85\%-100\%) at year $5,77.3 \%(63 \%-91 \%)$ at year 10 , and $66.8 \%$ $(50 \%-83 \%)$ at years 15 and 20 .

For the maxilla and mandible, Figs 5 to 7 show the Kaplan-Meier survival curves overall and for the vital and RCT groups. For the overall estimation (Fig 5), there was no significant difference $(P=.097)$ between the vital and RCT groups at year 20. The survival estimation in the maxilla was $88.7 \%(78 \%-99 \%)$ at year 5 , $73.4 \%(57 \%-90 \%)$ at year 10 , and $58.3 \%(38 \%-78 \%)$ at years 15 and 20. In the mandible, the survival estimation was $95.2 \%(90 \%-100 \%)$ at year $5,81.5 \%$ $(70 \%-93 \%)$ at year 10 , and $73.6 \%(60 \%-87 \%)$ at years 15 and 20.

Regarding the survival estimation for the vital group (Fig 6), no significant difference was found between the maxilla and mandible ( $P=.246)$. These survival estimates are mentioned above. The survival estimations for the RCT group (Fig 7) in the maxilla and mandible were significantly different $(P=.011)$. These survival estimates were mentioned earlier. Table 4 summarizes the results of Figs 3, 4, 6, and 7, revealing a significant difference in failures for the RCT group in the maxilla.

\section{Reasons for Failure}

The main reason for an irreversible complication was caries $(32.0 \%$ of failures). Loss of retention caused $12.0 \%$ of the irreversible complications. In $28.0 \%$ of failures, both caries and loss of retention were observed. The mean survival times for these groups of irreversible complications were 10.9 years for caries, 6.1 years for loss of retention, and 11.3 years for the combined group of caries and loss of retention. Fracture of

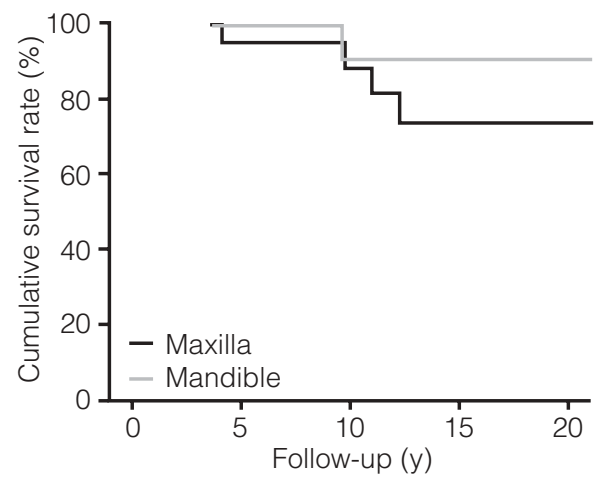

Fig 6 Kaplan-Meier survival curves for the maxilla and mandible in the vital group $(P=.246)$.

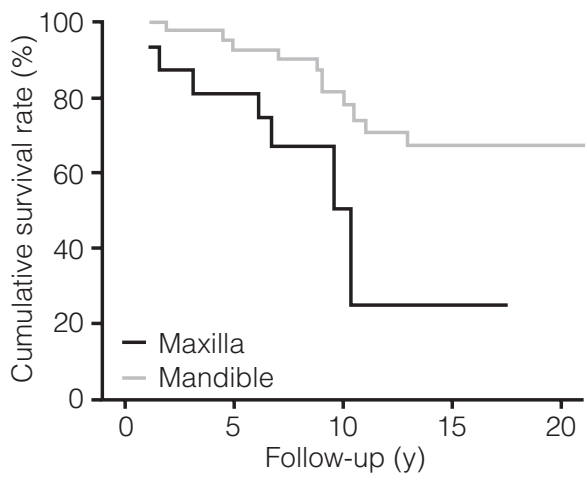

Fig 7 Kaplan-Meier survival curves for the maxilla and mandible in the RCT group $(P=.011)$.

the framework accounted for $12.0 \%$ of failures, and abutment fracture occurred in $8.0 \%$. Periodontal and apical problems both represented $4.0 \%$ of the irreversible complications.

Table 5 shows a cross-tabulation of the surviving restorations versus the failing restorations, with reversible complication as the dependent variable. In the surviving group, only $13.0 \%$ had a reversible complication, while in the failing group, $36.0 \%$ had a reversible complication. This was a significant difference $(P=.017)$.

These reversible complications were divided into an early reversible complication group (failure within 2 years) and a late reversible complication group (failure occurring after 2 years). For the failing restorations, the mean survival time of the early reversible complication group was 6.6 years, while the mean survival time of the late reversible complication group was 10.5 years, which is significantly different $(P=.036)$. 
Table 4 Twenty-Year Survival Rates (\%) in the Maxilla and Mandible for the Vital and RCT Groups

\begin{tabular}{llll}
\hline & Vital & RCT & $P$ \\
\hline Maxilla & 73.8 & $25.1^{*}$ & .007 \\
Mandible & 90.9 & 66.8 & .092 \\
$P$ & .246 & .011 & \\
\hline${ }^{*} 17-$ rear survival & & &
\end{tabular}

*17-year survival.

\section{Discussion}

Reported studies on treatment outcomes for different FDP designs tend to suggest consistent and favorable results. However, it must be acknowledged that numerous differences in the employed research designs demand a prudent interpretation of the published conclusions. Some of the key concerns and criticisms include the reconciliation of the research protocol with the following items: prospective versus retrospective protocols, duration of the observation period, oral site location, occlusal design considerations, patient behavioral considerations, etc.

However, reports such as the present study (and indeed most related publications), lend a certain amount of credence to the clinical decision-making process, and thus offer direction. It is in this context that this paper's results are offered for the reader's critical assessment.

In this particular study population, and within the framework of the employed evaluative criteria, there was no significant difference between overall outcomes in either dental arch, which is in agreement with some studies ${ }^{11,15,18,29}$ but in contrast with others. ${ }^{21,30,31}$

The overall survival of restorations in the vital group versus the RCT group revealed a significant difference at year 20 , with the use of RCT abutments leading to significantly more FDP failures. These results are comparable with some reported data ${ }^{10,21,22}$ but statistically different from others. ${ }^{19,29,32}$ Comparing these results with those from studies of full crowns and 3-unit FDPs at the same university ${ }^{29,33}$ showed no statistically significant difference between vital and RCT groups at years 18 and 20 . It can therefore be suggested that the use of an RCT abutment becomes a crucial concern in FDPs with 4 or more units. The quality of maxillary FDPs in the RCT group was inferior to that of vital FDPs in the maxilla and mandible. Vital FDPs in the
Table 5 Cross-Tabulation of Surviving Restorations Versus Failed Restorations, with Reversible Complications as the Dependent Variable*

\begin{tabular}{lccc}
\hline & \multicolumn{3}{c}{ Reversible complication (\%) } \\
\cline { 2 - 4 } Restorations & No & Yes & Total \\
\hline Surviving & $67(87.0)$ & $10(13.0)$ & 77 \\
Failed & $16(64.0)$ & $9(36.0)$ & 25 \\
\hline${ }^{*} P=017$ & &
\end{tabular}

${ }^{\star} P=.017$.

mandible had much better survival rates than vital FDPs in the maxilla and RCT FDPs in the mandible. In the present study group, a 4-unit FDP without RCT abutments in the mandible had the smallest number of irreversible complications. These results are confirmed by the same authors in 2 other similarly designed outcome studies. ${ }^{15,29}$

Whereas mechanical problems are, in general, more directly influenced by the clinician, biologic problems are less easily controlled and may be unrelated to the prosthetic treatment. However, biologic problems may be a consequence of the treatment procedures (pulpal problems) or may be influenced by the outline and gingival relation of the restorations (secondary caries, gingivitis, or periodontal destruction). The authors previously proposed ${ }^{15,29}$ hypothesis was that when more abutment teeth are used for the replacement of an equal number of missing teeth (ie, a lower pontic/ abutment ratio), the percentage of loss will be higher, sometimes with a low percentage of caries. In the study of FDPs with 3 units in function, ${ }^{29}$ the failure rate was $38.1 \%$ because of caries and $9.5 \%$ because of loss of retention. In the study of FDPs with 3 to 9 units in function (combined with a high pontic/abutment ratio), ${ }^{15}$ the failure rates were $22.2 \%$ because of caries and $15.3 \%$ because of loss of retention. In the current study, the failure rate because of caries was $32.0 \%$ and the failure rate because of loss of retention was $12.0 \%$. The authors are tempted to interpret this as a confirmation of the hypothesis. Moreover, these results endorse other studies that identify caries as the main cause of failure, ${ }^{7,15,20,22,23,32-35}$ while studies based on FDPs with more abutments in function for an equal replacement of missing teeth ${ }^{18,36-40}$ reported loss of retention as the main cause of failure. Caries detection was assumed to be a secondary problem caused by the loose retainer. 
Some authors reported on the mean life span of FDPs in relation to the reason for failure. Walton et al, ${ }^{10}$ Foster, ${ }^{41}$ and Valderhaug ${ }^{23}$ came to similar conclusions, namely that the mean life span with caries as the reason for failure was between 8.4 and 12 years, while for loss of retention, the mean life span was between 4.5 and 9 years. In the present study, the mean life span with caries as the reason for failure was 10.9 years, while for loss of retention, the mean life span was 6.1 years. Consequently, the combined loss of retention and caries group had a mean life span of 11.3 years, and it seems likely that the largest part of failures in this combined group will be caries. Thus, it can be concluded that caries failure accounted for $32.0 \%$ to $60.0 \%$ of failures. If loss of retention is the main cause of failure in prosthetic reconstructions with a low pontic/ abutment ratio, it may be suggested that caries is not the major problem due to the longer mean life span when caries causes failure. The results of the present and other surveys provide insights into compromised outcomes that result from caries, loss of retention, or both. ${ }^{15,29,33}$

In previous studies, ${ }^{15,33}$ the occurrence of a reversible complication had a predictive value for irreversible complications later on. This was confirmed by the results of the current study.

\section{Conclusions}

The estimated successful treatment outcome or survival rate of 4-unit FDPs replacing 2 adjacent missing teeth at year 20 was $68.3 \%$. There was a statistically significant difference for the survival between the vital and root canal-treated groups, but not between the maxilla and mandible. Caries and loss of retention were the main reasons for failure, accounting for $72 \%$ of failures combined. Caries accounted for $32.0 \%$ to $60.0 \%$ of failures. The mean life span was 10.9 years with caries as the reason for failure and 6.1 years with loss of retention as the main reason for failure. More retainers in function will probably increase the risk of loss of retention and sometimes the loss of the FDP. A 4-unit FDP without RCT abutments in the mandible showed the smallest number of irreversible complications. Occurrence of a reversible complication appears to be a predictive factor for an irreversible complication later on. A reversible complication within the first 2 years was observed to lead to an early irreversible complication, with a mean survival time of 6.6 years.

\section{Acknowledgments}

The authors are very thankful to Veerle Decock and Frank Herrebout from the former Department of Fixed Prosthodontics and Periodontology, Dental School, Faculty of Medicine and Health Sciences, Ghent University, Belgium, for their valuable help.

\section{References}

1. Belser U, Buser D, Hess D, Schmid B, Bernard J-P, Lang NP. Aesthetic implant restorations in partially edentulous patientsA critical appraisal. Periodontol 2000 1998;17:132-150.

2. Ingber JS, Rose LF, Coslet JG. The "biologic width"-A concept in periodontics and restorative dentistry. Alpha Omegan 1977; 70:62-65.

3. Chang M, Wennström JL, Ödman P, Andersson B. Implantsupported single-tooth replacements compared to contralateral natural teeth. Clin Oral Implants Res 1999;10:185-194.

4. Camargo P, Melnick P, Kenney E. The use of free gingival grafts for esthetic purposes. Periodontology 2000 2001;27:72-96.

5. Donovan T, Cho G. Predictable esthetics with metal-ceramic and all-ceramic crowns: The critical importance of soft-tissue management. Periodontol 2000 2001;27:121-130.

6. Chee W. Provisional restorations in soft tissue management around dental implants. Periodontol 2000 2001;27:139-147.

7. Goodacre C, Bernal G, Rungcharassaeng K, Kan J. Clinical complications in fixed prosthodontics. J Prosthet Dent 2003;90:31-41.

8. Goodacre C, Bernal G, Rungcharassaeng K, Kan J. Clinical complications with implants and implant prostheses. J Prosthet Dent 2003;90:121-132.

9. Schwartz NL, Whitsett LD, Berry TG, Steward JL. Unserviceable crowns and fixed partial dentures: Life-span and causes for loss of serviceability. J Am Dent Assoc 1970;81:1396-1401.

10. Walton J, Gardner F, Agar J. A survey of crown and fixed partial denture failures: Length of service and reason for replacement. J Prosthet Dent 1986;56:416-421.

11. Kerschbaum Th, Gaa M. Longitudinale Analyse von festsitzendem Zahnersatz privatversicherter Patienten. Dtsch Zahnärztl Z 1987; 42:345-351.

12. Kerschbaum Th, Leempoel P. Kronen und Brücken. In: Meiners H (ed). Fortschritte der zahnärztlichen Prothetik und Werkstoffkunde, vol IV. München: Hanser, 1989:109-136.

13. Foster $\mathrm{L}$. The relationship between failure and design in conventional bridgework from general dental practice. J Oral Rehabil 1991;18:491-495.

14. Fayyad MA, Al-Rafee MA. Failure of dental bridges: III-effect of some technical factors. J Oral Rehabil 1996;23:675-678.

15. De Backer H, Van Maele G, De Moor N, Van den Berghe L, De Boever J. A 20-year retrospective survival study of fixed partial dentures. Int J Prosthodont 2006;19:143-153.

16. Walton T. An up to 15-year longitudinal study of 515 metal-ceramic FPDs: Part 1. Outcome. Int J Prosthodont 2002;15:439-445.

17. Reuter JE, Brose MO. Failures in full crown retained dental bridges. Br Dent J 1984;157:61-63.

18. Palmqvist S, Swartz B. Artificial crowns and fixed partial dentures 18 to 23 years after placement. Int J Prosthodont 1993;6:279-285.

19. Leempoel P, Käyser A, Van Rossum G, De Haan A. The survival rate of bridges. A study of 1674 bridges in 40 Dutch general practices. J Oral Rehabil 1995;22:327-330.

20. Sundh B, Ödman P. A study of fixed prosthodontics performed at a university clinic 18 years after insertion. Int J Prosthodont 1997;10:513-519.

21. Palmqvist S, Söderfeldt B. Multivariate analyses of factors influencing the longevity of fixed partial dentures, retainers and abutments. J Prosthet Dent 1994;71:245-250.

22. Holm C, Tidehag P, Tillberg A, Molin M. Longevity and quality of FPDs: A retrospective study of restorations 30,20 , and 10 years after insertion. Int J Prosthodont 2003;16:283-289.

23. Valderhaug J. A 15-year clinical evaluation of fixed prosthodontics. Acta Odontol Scand 1991;49:35-40.

24. Creugers N, Käyser A, Van 't Hof M. A meta-analysis of durability data on conventional fixed bridges. Community Dent Oral Epidemiol 1994;22:448-452. 
25. Scurria M, Bader J, Shugars D. Meta-analysis of fixed partial denture survival: Prostheses and abutments. J Prosthet Dent 1998; 79:459-464.

26. Tan K, Pjetursson BE, Lang NP, Chan ES. A systematic review of the survival and complication rates of fixed partial dentures (FPDs) after an observation period of at least 5 years. III. Conventional FPDs. Clin Oral Implants Res 2004;15:654-666.

27. Kaplan EL, Meier P. Nonparametric estimation from incomplete observations. J Am Stat Assess 1958;53:457-481.

28. Kalbfleisch JD, Prentice RL. The Statistical Analysis of Failure Time Data. New York: John Wiley \& Sons, 1980.

29. De Backer H, Van Maele G, De Moor N, Van den Berghe L. Singletooth replacement: Is a 3-unit fixed partial denture still an option? A 20-year retrospective study. Int J Prosthodont 2006;19:567-573.

30. Kerschbaum T, Paszyna C, Klapp S, Meyer G. Verweilzeit-und Risikofaktorenanalyse von festsitzendem Zahnersatz. Dtsch Zahnärztl Z 1991;46:20-24.

31. Hochman N, Mitelman L, Hadani PE, Zalkind M. A clinical and radiographic evaluation of fixed partial dentures (FPDs) prepared by dental school students: A retrospective study. J Oral Rehabil 2003; 30:165-170.

32. Valderhaug J, Jokstad A, Ambjornsen E, Norheim W. Assessment of the periapical and clinical status of crowned teeth over 25 years. J Dent 1997;25:97-105
33. De Backer H, Van Maele G, De Moor N, Van den Berghe L, De Boever J. An 18-year retrospective survival study of full crowns with or without posts. Int J Prosthodont 2006;19:136-142.

34. Randow K, Glantz P-O, Zöger B. Technical failures and some related clinical complications in extensive fixed prosthodontics. Acta Odontol Scand 1986;44:241-255.

35. Libby G, Arcuri M, LaVelle W, Hebl L. Longevity of fixed partial dentures. J Prosthet Dent 1997;78:127-131.

36. Glantz PO, Ryge G, Jendresen M, Nilner K. Quality of extensive fixed prosthodontics after five years. J Prosthet Dent 1984;52: 475-479.

37. Karlsson S. A clinical evaluation of fixed bridges, 10 years following insertion. J Oral Rehabil 1986;13:423-432.

38. Karlsson S. Failures and length of service in fixed prosthodontics after long-term function. Swed Dent J 1989;13:185-192.

39. Glantz PO, Nilner K, Jendresen M, Sundberg H. Quality of fixed prosthodontics after 15 years. Acta Odontol Scand 1993;51: 247-252.

40. Lindquist $E$, Karlsson S. Success rate and failures for fixed partial dentures after 20 years of service: Part 1. Int J Prosthodont 1998; 11:133-138

41. Foster L. Failed conventional bridgework from general dental practice: Clinical aspects and treatment needs of 142 cases. Br Dent J 1990;10:199-201.

\section{A retrospective evaluation of a treatment protocol for dental implant periapical lesions: Long-term results of $39 \mathrm{implant}$ apicoectomies}

The objectives of this retrospective clinical study were to describe a treatment protocol for treating implants with periapical lesions and to present the results of such treatment. Thirty-five dental implant patients (mean age: 58.3 years) previously treated in a private prosthodontic practice were identified with an implant periapical lesion. There were a total of 39 lesions identified either radiographically (radiolucency), by clinical observation (swelling, suppuration, fistula), or by a combination of both. Twenty-six of 39 lesions (66.7\%) showed clinical signs of infection. Patients were excluded if the lesion had spread coronally to the crest of the alveolar ridge, creating oral communication with the lesion, or had caused implant mobility or failure. Using an intraoral approach, local anesthesia with a combination of bupivacaine hydochloride and epinephrine and lidocaine hydrochloride was administered, followed by elevation of a flap facial to the implant site, exposing the bone. A periapical film was used to measure the abscess in the area of the implant apical lesion. A carbide bur in a high-speed drill was used to open a window in the bone, and a curette was used to debride the bony defect. Biopsy samples of excised tissue were sent for histologic analysis. A carbide bur was then used to remove the affected portion of the implant. An average length of $3.6 \mathrm{~mm}$ (range: 2 to $6 \mathrm{~mm}$ ) of implant was removed. The area was then thoroughly debrided and irrigated with tetracycline/saline solution. In most cases, Bio-Oss bovine bone was used to graft the defect, with or without the use of a Bio-Gide membrane. The remaining patients received neither bone grafting nor membranes prior to primary closure. Postoperative antibiotics and pain medication were prescribed for all patients. Panoramic and periapical radiographs were obtained following treatment. Seventeen treated implants were in the maxilla: 9 anterior and 8 posterior. The remaining 22 treated implants were in the mandible: 11 anterior and 11 posterior. Of the implants, $51.28 \%$ were placed in type 3 bone, $2.56 \%$ in type $1,33.33 \%$ in type 2 , and $12.8 \%$ in type 4 . The average length of the implants treated was $15.5 \mathrm{~mm}$. The majority of apical lesions appeared within the first 2 years after implant placement. Follow-up time averaged 4.54 years (range: 0.84 to 15.02 years). Thirty-eight of 39 implants treated remained stable and in clinical function, with no signs of recurrence after clinical and radiographic examination, yielding a cumulative survival rate of $97.4 \%$. The 1 implant that failed after treatment was previously placed in type 4 bone in the anterior mandible in a 53-year-old man who smoked at least 2 packs of cigarettes per day. Histology reports from 37 of 39 sites showed an infiltrate of inflammatory cells in a stroma of immature collagen fibers interspersed by active fibrocytes and numerous dilated capillaries. None of the biopsies demonstrated malignant features. The authors recommended that it is crucial to treat the implant before the lesion spreads coronally, since a channel would exist between the oral cavity and osseous environment for bacterial migration should the lesion reach the portion of the implant that has an internal screw thread. It would be interesting to find out what would be the minimum length of implant required or the critical size of the periapical lesion before this mode of treatment can be instituted.

Balshi SF, Wolfinger GJ, Balshi TJ. Int J Oral Maxillofac Implants 2007;22:267-272. References: 26. Reprints: Mr Stephen F. Balshi, Prosthodontics Intermedica, 467 Pennsylvania Ave, Suite 201, Fort Washington, PA 19034. E-mail: balshi2@aol.com—Elvin W.J. Leong, Singapore 